\title{
Multisymplectic Geometry and Multisymplectic Preissman Scheme for the KP Equation *
}

\author{
Tingting Liu, Menzhao Qin \\ CCAST(World Laboratory), \\ PoBox 8730, Beijing 100080, China \\ Institute of Computational Mathematics, \\ Academy of Mathematics and Systems Sciences, \\ Chinese Academy of Sciences, \\ PoBox 2719, Beijing, 100080, China \\ ttliu@lsec.cc.ac.cn, qmz@lsec.cc.ac.cn
}

\begin{abstract}
The multisymplectic structure of the KP equation is obtained directly from the variational principal. Using the covariant De Donder-Weyl Hamilton function theories, we reformulate the KP equation to the multisymplectic form which proposed by Bridges. From the multisymplectic equation, we can derive a multisymplectic numerical scheme of the KP equation which can be simplified to multisymplectic forty-five points scheme.
\end{abstract}

\section{Introduction}

The generalized Kadomtsev-Petviashvili (GKP) equation is

$$
\left(u_{t}+\partial_{x} f(u)+u_{x x x}\right)_{x}+\sigma u_{y y}=0 \quad(t>0,-\infty<x, y<\infty)
$$

where $\sigma$ is a constant, $f(u)$ is some smooth function. The usually KP equation occurs for $f(u)$ quadratic and it is regarded as a two-dimensional generalization of the Korteweg-de Vries $(\mathrm{KdV})$ equation. It describes the evolution of long water waves of small amplitude if they are weakly two-dimensional. In the case of $f(u)=3 u^{2}$ and $\sigma=-3$, Equation (1.1) is usually called the KPI equation, whereas the KPII equation with $f(u)=3 u^{2}$ and $\sigma=3$. As a soliton equation important from analytical and numerical point of view, the KP equation is one of the few known completely integrable equations in the multidimensional soliton equations. Thus, in the last few years, considerable attention has been paid to the KP equation. Although considerable interest has been focused on the $\mathrm{KP}$ equation, the numerical scheme analysis literature for the KP equation is extremely small. As far as we are aware of, Katsis proposed explicit finite difference method [15], the results of evolution of lump solution for the KP equation was given by A.A. Minzoni

${ }^{*}$ Supported by the Special Funds for Major State Basic Research Projects, G 1999, 032800. 
[17], X.P. Wang et. studied the instability of a generalized KP equation [16], B.F. Feng and T. Mitsui took the linearized implicit method to the KP equation [13].

In this paper, we try describe KP equation in the language of multisymplectic geometry. Recently, for first order field theory, i.e., the Lagrangian density depends on the state variables and their first order derivatives, Marsden, Patrix and Shkoller [7] derived numerical methods for the first order field theories. However the lagrangian density of the KP equation is not first-order, therefore MPS theory can not be applied directly. In [10], the authors focus their attention on the KdV equation whose Langrangian density is second order. The langrangian density of the KP equation is truly third-order. In this paper, we give the multisymplectic structure of the KP equation directly from the variational principal. In [5], the author proposed the Cartan form is not necessarily unique, we find it was caused by higher-order mixed multiple integral in using Stokes' formula in actually calculus variation.

In Lagrange mechanics, we know the Euler-Lagrange equation can be write as

$$
\frac{d}{d t} \frac{\partial L}{\partial \dot{q}^{i}}-\frac{\partial L}{\partial q^{i}}=0
$$

Taking the Legandre transform of Lagrange density $L: p^{i}=\frac{\partial L}{\partial \dot{q}^{i}}$, we can rewrite equation (1.2) as regular Hamilton equation

$$
\left\{\begin{array}{l}
\frac{d p^{i}}{d t}=-\frac{\partial H}{\partial q^{i}} \\
\frac{d q^{i}}{d t}=\frac{\partial H}{\partial p^{i}}
\end{array}\right.
$$

where $H=p^{i} \dot{q}^{i}-L\left(q^{i}, \dot{q}^{i}, t\right)$. With the covariant De Donder-Weyl Hamilton function theories [18], we can reformulate the partial differential equation to the following form

$$
\left\{\begin{array}{l}
\frac{\partial H}{\partial \pi_{i}^{\mu}}=\partial_{\mu} q^{i} \\
\frac{\partial H}{\partial q^{i}}=-\partial_{\mu} \pi_{i}^{\mu}
\end{array}\right.
$$

where $\pi_{i}^{\mu}=\frac{\partial L}{\partial \partial_{\mu} q^{i}}$. According to this methods, we can rewrite KP equation to the multisymplectic form that introduced by Bridges [3].

Multisymplectic equation have the important multisymplectic conservation laws. In the numerical study, we also hope that the numerical approximations can preserve the multisymplectic conservation laws. Similar to the method [9], we show that the Preissman scheme is a multisymplectic scheme for the KP equation. Though the Preissman scheme is multisymplectic, it need more computational memory, so we reduce it to a multisymplectic forty-five points scheme. Using the forty-five points scheme, we get some numerical results on soliton and solitary waves over long time intervals.

In section 2, we describe the multisymplectic geometry of the KP equation entirely in the framework of the variational principal. Section 3 is devoted to the analysis of multisymplectic Preissman scheme and reduce it to a multisymplectic forty-five points scheme. In section 4, some numerical results on soliton and solitary waves over long time intervals be given. 


\section{Multisymplectic Geometry of the KP Equation}

We now review some aspects of multisymplectic geometry.

Let $X$ be an orientable $(n+1)$-dimensional parameter space ( which is usually space time ) and let $\pi_{X Y}: Y \rightarrow X$ be a fiber over $X$. Section $\varphi: X \rightarrow Y$ of this covariant configuration bundle is the physical fields. Coordinates on $X$ are denoted by $x^{\mu}, \mu=$ $1,2, \cdots, n, 0$. In general, $x^{0}$ denotes the time coordinate. The parameter $n$ denotes the number of spatial variables. In this paper, we just discuss the case of $n>0$. Adapted coordinates on $Y$ are $y^{A}$ along the fibers $Y_{x}=\pi_{X Y}^{-1}(x), x \in X, A=1,2, \cdots, N$. $N$ denote the fibers dimensions. Consider a $k^{t h}$ order lagrangian density $L$, viewed as a fiber-preserving map $L: J^{k} Y \rightarrow \wedge^{n+1} X . J^{k}(Y)$ denote the $k^{\text {th }}$-order jet bundle over $Y$ which can be induced by $J^{1}\left(\cdots\left(J^{1}(Y)\right)\right)$. We let $T_{x} X$ denote the tangent space of $X$ at $x$. Denote the derivative of the map $\pi_{X Y}$ in the direction $w$ by $T_{\pi_{X Y}} \cdot w$.

At first, we introduce the first jet bundle.

Definition 2.1 The first jet bundle over $Y$ is a fiber bundle denoted by $J^{1}(Y)$ whose fiber over $y \in Y_{x}=\pi_{X Y}^{-1}(x), x \in X$ consists of those linear mappings $\gamma: T_{x} X \rightarrow T_{y} Y$ satisfying

$$
T_{\pi_{X Y}} \circ \gamma=\text { Identity }
$$

If $\varphi: X \rightarrow Y$ is a section of $Y, j^{1}(\varphi)$ is a section of $J^{1}(Y)$ and in coordinates, $j^{1}(\varphi)$ is given by

$$
\left(x^{\mu}, \varphi^{A}\left(x^{\mu}\right), \partial_{\mu} \varphi^{A}\left(x^{\mu}\right)\right), \quad \mu=1,2, \cdots, n, 0,
$$

Similarly, higher order jet bundle $J^{m}(Y)$ is defined by $J^{1}\left(J^{m-1}(Y)\right)$.

Definition 2.2 The $k^{\text {th }}$-order jet bundle over $Y$ is a fiber bundle denoted by $J^{k}(Y)$ whose fiber over $\gamma \in J^{k-1}(Y)_{y}, y \in Y$ consists of those linear mappings $s: T_{x} X \rightarrow T_{\gamma} J^{k-1}(Y)$ satisfying

$$
T_{\pi_{X, J} k-1_{Y}} \circ s=\text { Identity. }
$$

We let $j^{k}(\varphi)=j^{1}\left(\cdots\left(j^{1}(\varphi)\right): x \rightarrow T_{x} j^{k-1}(\varphi)\right.$ denote $k^{\text {th }}$-order jet prolongation of the section $\varphi: X \rightarrow Y$, in which $j^{k-1}(\varphi)$ is a section of jet bundle $J^{k-1}(Y)$. Thus, $j^{k}(\varphi)$ is given in coordinates

$$
\left(x^{\mu}, \varphi^{A}\left(x^{\mu}\right), \partial_{\mu} \varphi^{A}\left(x^{\mu}\right), \cdots, \partial_{\mu_{n}} \partial_{\mu_{n-1}} \cdots \partial_{\mu_{0}} \varphi^{A}\left(x^{\mu}\right)\right),
$$

Given a $k^{t h}$-order lagrangian density $L: J^{k} Y \rightarrow \wedge^{n+1} x$, the basis geometric object in the classical calculus of variations is the $(n+1)$-form $\theta_{L}$ on $J^{2 k-1}(Y)$, which was called the Cartan form.

The KP equation can be written as

$$
\left(2 u_{t}+6 u u_{x}+u_{x x x}\right)_{x}+\sigma u_{y y}=0 .
$$

The 2 multiplying $u_{t}$ is added for notational convenience, it can be eliminated by scaling $t$. In this paper, we consider the KPI equation.

To put the KP equation in the variational frame work, we let $\varphi_{x x}=u$, then $\varphi$ satisfies equation:

$$
2 \varphi_{x x x t}+6 \varphi_{x x} \varphi_{x x x x}+6 \varphi_{x x x}^{2}+\varphi_{x x x x x x}+\sigma \varphi_{x x y y}=0
$$


The search for a variational principle is equivalent to the inverse problem of the calculus of variations, i.e., the existence and formulation of variational principles for systems of nonlinear partial differential equations. The existence of a variational principle for a differential equation is equivalent to determining whether or not an operator is a potential operator. According to Vainberg theorem [2], in order that operator $N$ be potential operator, to summarize, it is necessary and sufficient that the Gateau derivative of the operator $N$ is symmetry. The theorem stated in terms of the Gateau derivative, but we assume that the Frechet derivative exists in application. Let $N$ is an operator which defined in an appropriate function space $E$ (typically $E$ is Banach space), then $N$ be potential operator if $N_{u}^{\prime}=\tilde{N}_{u}^{\prime}$, where

$$
N_{u}^{\prime} \varphi=\lim _{\varepsilon \rightarrow 0} \frac{N(u+\varepsilon \varphi)-N(u)}{\varepsilon}=\left.\left[\frac{\partial}{\partial \varepsilon} N(u+\varepsilon \varphi)\right]\right|_{\varepsilon=0}
$$

and $\tilde{N}_{u}^{\prime}$ is the adjoint operator of $N_{u}^{\prime}$. If the operator is a potential operator, the potential $F$ is given by

$$
F=\int u \int_{0}^{1} N(\lambda u) d \lambda d V
$$

where $\int d V$ represents integration over the physical domain and $\int_{0}^{1} d \lambda$ represents integration over the scalar variable $\lambda$. We test the operator

$$
N(\varphi)=2 \varphi_{x x x t}+6 \varphi_{x x} \varphi_{x x x x}+6 \varphi_{x x x}^{2}+\varphi_{x x x x x x}+\sigma \varphi_{x x y y}
$$

and find $N_{\varphi}^{\prime}=\tilde{N}_{\varphi}^{\prime}$. Hence,

$$
\begin{aligned}
F(\varphi) & =\int \varphi \int_{0}^{1} N(\lambda \varphi) d \lambda d V \\
& =\int \varphi \int_{0}^{1}\left[2 \lambda \varphi_{x x x t}+6 \lambda^{2} \varphi_{x x} \varphi_{x x x x}+6 \lambda^{2} \varphi_{x x x}^{2}+\lambda \varphi_{x x x x x x}+\sigma \lambda \varphi_{x x y y}\right] d \lambda d V \\
& =\int \varphi\left(\varphi_{x x x t}+2 \varphi_{x x x}^{2}+2 \varphi_{x x} \varphi_{x x x x}+\frac{1}{2} \varphi_{x x x x x x}+\frac{\sigma}{2} \varphi_{x x y y}\right) d V
\end{aligned}
$$

To obtain $F(u)$ in a more familiar form, integrate by part and discard the integration over the boundary since it has nothing with the Lagrangian density, then we get potential

$$
F(\varphi)=\int\left(\varphi_{x x} \varphi_{x t}-\frac{1}{2} \varphi_{x x x}^{2}+\frac{\sigma}{2} \varphi_{x y}^{2}+\varphi_{x x}^{3}\right) d V .
$$

So we can determined that the Lagrangian density for equation (2.2) is

$$
L\left(j^{3}(\varphi)\right)=\left(\varphi_{x x} \varphi_{x t}-\frac{1}{2} \varphi_{x x x}^{2}+\frac{\sigma}{2} \varphi_{x y}^{2}+\varphi_{x x}^{3}\right) d x \wedge d y \wedge d t
$$

Corresponding to Lagrangian density $L\left(j^{3}(\varphi)\right)$, the action function is defined as following:

$$
S(\varphi)=\int_{U} L\left(j^{3}(\varphi)\right), \quad U \text { is a open set of } X .
$$

Let $\mathrm{G}$ be the Lie group of $\pi_{X Y}$ bundle automorphisms $\eta_{Y}$ covering $\eta_{X}$. Denote $\eta_{Y}^{\lambda}$ an smooth path in $\mathrm{G}$ such that

$$
\bar{\varphi}=\eta_{Y}^{\lambda} \circ \varphi \circ\left(\eta_{X}^{\lambda}\right)^{-1} .
$$


The vector field of $\eta_{Y}^{\lambda}$ is

$$
V=\left.\frac{d}{d \lambda}\right|_{\lambda=0} \bar{\varphi}=\left[\begin{array}{c}
V^{x} \\
V^{y} \\
V^{t} \\
V^{\varphi}
\end{array}\right] .
$$

We say that $\varphi$ is a extremum of $S$ is

$$
\left.\frac{d}{d \lambda}\right|_{\lambda=0} S(\bar{\varphi})=0
$$

Now we consider the variation

$$
\left.\frac{d}{d \lambda}\right|_{\lambda=0} S(\bar{\varphi})=\left.\frac{d}{d \lambda}\right|_{\lambda=0} \int_{\eta_{X}^{\lambda} U}\left(\bar{\varphi}_{\overline{x x}} \bar{\varphi}_{\bar{x} \bar{t}}-\frac{1}{2} \bar{\varphi}_{\overline{x x x}}^{2}+\frac{\sigma}{2} \bar{\varphi}_{\overline{x y}}^{2}+\bar{\varphi}_{\overline{x x}}^{3}\right) d \bar{x} \wedge d \bar{y} \wedge d \bar{t}
$$

where

$$
\eta_{X}^{\lambda}\left[\begin{array}{l}
x \\
y \\
t
\end{array}\right]=\left[\begin{array}{l}
\bar{x} \\
\bar{y} \\
\bar{t}
\end{array}\right]
$$

A direct computation shows

$$
\left.\frac{d}{d \lambda}\right|_{\lambda=0} S(\bar{\varphi})=I_{1}+I_{2}
$$

in which

$$
\begin{aligned}
& I_{1}=\int_{U}-\left(2 \varphi_{x x x t}+6 \varphi_{x x} \varphi_{x x x x}+6 \varphi_{x x x}^{2}+\varphi_{x x x x x x}+\sigma \varphi_{x x y y}\right) \\
& \left(V^{\varphi}-\varphi_{x} V^{x}-\varphi_{y} V^{y}-\varphi_{t} V^{t}\right) d x \wedge d y \wedge d t, \\
I_{2}= & \int_{\partial U}\left[\left(\varphi_{x x} \varphi_{x t}-\frac{1}{2} \varphi_{x x x}^{2}+\frac{\sigma}{2} \varphi_{x y}^{2}+\varphi_{x x}^{3}\right) V^{t}\right. \\
& \left.+\varphi_{x x}\left(V_{x}^{\varphi}-\varphi_{x} V_{x}^{x}-\varphi_{y} V_{x}^{y}-\varphi_{t} V_{x}^{t}-\varphi_{x x} V^{x}-\varphi_{x y} V^{y}-\varphi_{x t} V^{t}\right)\right] d x \wedge d y \\
& {\left[-\left(\varphi_{x x} \varphi_{x t}-\frac{1}{2} \varphi_{x x x}^{2}+\frac{\sigma}{2} \varphi_{x y}^{2}+\varphi_{x x}^{3}\right) V^{y}\right.} \\
& \left.+\sigma \varphi_{x y}\left(V_{x}^{\varphi}-\varphi_{x} V_{x}^{x}-\varphi_{y} V_{x}^{y}-\varphi_{t} V_{x}^{t}-\varphi_{x x} V^{x}-\varphi_{x y} V^{y}-\varphi_{x t} V^{t}\right)\right] d x \wedge d t \\
+ & \left(\varphi_{x x} \varphi_{x t}-\frac{1}{2} \varphi_{x x x}^{2}+\frac{\sigma}{2} \varphi_{x y}^{2}+\varphi_{x x}^{3}\right) V^{x} \\
+ & \left(\varphi_{x t}+3 \varphi_{x x}^{2}+\varphi_{x x x}\right)\left(V_{x}^{\varphi}-\varphi_{x} V_{x}^{x}-\varphi_{y} V_{x}^{y}-\varphi_{t} V_{x}^{t}-\varphi_{x x} V^{x}\right. \\
& \left.-\varphi_{x y} V^{y}-\varphi_{x t} V^{t}\right)-\varphi_{x x x}\left(V_{x x}^{\varphi}-\varphi_{x} V_{x x}^{x}-2 \varphi_{x x} V_{x}^{x}-\varphi_{y} V_{x x}^{y}\right. \\
& \left.-2 \varphi_{x y} V_{x}^{y}-\varphi_{t} V_{x x}^{t}-2 \varphi_{x t} V_{x}^{t}-\varphi_{x x y} V^{y}-\varphi_{x x t} V^{t}-\varphi_{x x x} V^{x}\right) \\
+ & \left.\left(-2 \varphi_{x x t}-6 \varphi_{x x} \varphi_{x x x}-\sigma \varphi_{x y y}-\varphi_{x x x x x}\right)\left(V^{\varphi}-\varphi_{x} V^{x}-\varphi_{y} V^{y}-\varphi_{t} V^{t}\right)\right] d y \wedge d t .
\end{aligned}
$$

$\operatorname{In}[5]$, the author introduced the Lepagean equivalents which is a generalization of the Poincare-Cartan form and proposed the Carton form is not necessarily unique for higher order. The author also pointed out that every Lagrangian density has a Lepagean equivalent on $J^{2 k-1} Y$. It turns out that Lepagean equivalents exists on jet bundles of order $2 k-1$ or higher, but not necessarily on jet bundle of lower order. The author presented that: As being the principal part of a Lepagean equivalents, the Cartan form 
always exist and are typically nonunique unless $k=1$. we find it is caused by the higherorder mixed multiple integral in using Stokes' formula in actually calculus of variations. By $I_{2}$, we can define a Cartan form

$$
\begin{aligned}
\theta_{L}= & \left(\frac{1}{2} \varphi_{x x x}^{2}-\frac{\sigma}{2} \varphi_{x y}^{2}+2 \varphi_{x} \varphi_{x x t}+6 \varphi_{x} \varphi_{x x} \varphi_{x x x}+\sigma \varphi_{x} \varphi_{x y y}+\varphi_{x} \varphi_{x x x x x}\right. \\
& \left.-\varphi_{x x} \varphi_{x t}-2 \varphi_{x x}^{3}-\varphi_{x x} \varphi_{x x x x}\right) d x \wedge d y \wedge d t \\
& +\left(-2 \varphi_{x x t}-6 \varphi_{x x} \varphi_{x x x}-\sigma \varphi_{x y y}-\varphi_{x x x x x}\right) d \varphi \wedge d y \wedge d t \\
& +\left(\varphi_{x t}+3 \varphi_{x x}^{2}+\varphi_{x x x x}\right) d \varphi_{x} \wedge d y \wedge d t-\varphi_{x x x} d \varphi_{x x} \wedge d y \wedge d t \\
& -\sigma \varphi_{x y} d \varphi_{x} \wedge d x \wedge d t+\varphi_{x x} d \varphi_{x} \wedge d x \wedge d y
\end{aligned}
$$

Since

$$
\begin{aligned}
& j^{5}(\varphi)^{*} d x=d x, \quad j^{5}(\varphi)^{*} d y=d y, \quad j^{5}(\varphi)^{*} d t=d t \\
& j^{5}(\varphi)^{*} d \varphi=\varphi_{x} d x+\varphi_{y} d y+\varphi_{t} d t, \\
& j^{5}(\varphi)^{*} d \varphi_{x}=\varphi_{x x} d x+\varphi_{x y} d y+\varphi_{x t} d t, \\
& j^{5}(\varphi)^{*} d \varphi_{x x}=\varphi_{x x x} d x+\varphi_{x x y} d y+\varphi_{x x t} d t,
\end{aligned}
$$

we have

$$
\left.I_{2}=\int_{\partial U} j^{5}(\varphi)^{*}\left(j^{5}(V)\right\rfloor \theta_{L}\right)
$$

here $j^{5}(V)$ is the jet prolongation of the vector field $V$ [8]. The multisymplectic form is the 4 -form $\Omega_{L}=-d \theta_{L}$. Form $\theta_{L}$ defines a multisymplectic structure on jet bundle $J^{5}(Y)$.

Now, we consider the Euler-Lagrange equation for the action function $S(\varphi)$.

Since $L\left(j^{3}(\bar{\varphi})\right)=j^{5}(\bar{\varphi})^{*} \theta_{L}$, we have

$$
\begin{aligned}
\left.\frac{d}{d \lambda}\right|_{\lambda=0} \int_{\eta_{X}^{\lambda} U} L\left(j^{3}(\bar{\varphi})\right) & =\left.\frac{d}{d \lambda}\right|_{\lambda=0} \int_{\eta_{X}^{\lambda} U} j^{5}(\bar{\varphi})^{*} \theta_{L} \\
& =\left.\frac{d}{d \lambda}\right|_{\lambda=0} \int_{\eta_{X}^{\lambda} U} j^{5}\left(\eta_{Y}^{\lambda} \circ \varphi \circ\left(\eta_{X}^{\lambda}\right)^{-1}\right)^{*} \theta_{L} \\
& =\left.\frac{d}{d \lambda}\right|_{\lambda=0} \int_{\eta_{X}^{\lambda} U}\left(\left(\eta_{X}^{\lambda}\right)^{-1}\right)^{*} j^{5}(\varphi)^{*} j^{5}\left(\eta_{Y}^{\lambda}\right)^{*} \theta_{L} \\
& =\left.\frac{d}{d \lambda}\right|_{\lambda=0} \int_{U} j^{5}(\varphi)^{*} j^{5}\left(\eta_{Y}^{\lambda}\right)^{*} \theta_{L} \\
& =\int_{U} j^{5}(\varphi)^{*} £_{j^{5}(V)} \theta_{L}
\end{aligned}
$$

in which symbol $£$ denotes the Lie derivative.

By the Cartan's Magic formula [6]

$$
\left.\left.£_{j^{5}(V)} \theta_{L}=-j^{5}(V)\right\rfloor \Omega_{L}+d\left(j^{5}(V)\right\rfloor \theta_{L}\right)
$$

We can obtain

$$
\left.\left.\left.\frac{d}{d \lambda}\right|_{\lambda=0} S(\bar{\varphi})=-\int_{U} j^{5}(\varphi)^{*}\left(j^{5}(V)\right\rfloor \Omega_{L}\right)+\int_{\partial U} j^{5}(\varphi)^{*}\left(j^{5}(V)\right\rfloor \theta_{L}\right)
$$

If $V$ is a vector field with compact support, we have

$$
\left.\int_{\partial U} j^{5}(\varphi)^{*}\left(j^{5}(V)\right\rfloor \theta_{L}\right)=0
$$

Hence, a necessary condition for $\varphi$ to be an extremum is that

$$
\left.\int_{U} j^{5}(\varphi)^{*}\left(j^{5}(V)\right\rfloor \Omega_{L}\right)=0
$$


for any $V$ with compact support. By compute the integral and obtain that

$$
\begin{aligned}
\left.j^{5}(\varphi)^{*}\left(j^{5}(V)\right\rfloor \Omega_{L}\right)= & \left(2 \varphi_{x x x t}+6 \varphi_{x x} \varphi_{x x x x}+6 \varphi_{x x x}^{2}+\varphi_{x x x x x x}\right. \\
& \left.+\sigma \varphi_{x x y y}\right)\left(V^{\varphi}-\varphi_{x} V^{x}-\varphi_{y} V^{y}-\varphi_{t} V^{t}\right)
\end{aligned}
$$

Remark: We can get the Euler-lagrange equation from the vertical variation $V^{\varphi}$, and the $V^{x}$ and $V^{y}$ directions horizontal variations gives the law of conservation of momentum. The law of conservation of energy can be obtained along time-direction horizontal variation.

Taking the $\pi_{X Y}$-vertical vector field $V$ and using the standard method from the calculus of variations, we obtain that $\varphi$ satisfies

$$
2 \varphi_{x x x t}+6 \varphi_{x x} \varphi_{x x x x}+6 \varphi_{x x x}^{2}+\varphi_{x x x x x x}+\sigma \varphi_{x x y y}=0
$$

i.e., the equation (2.2). So, for any vector field $V$,

$$
\left.j^{5}(\varphi)^{*}\left(j^{5}(V)\right\rfloor \Omega_{L}\right)=0
$$

holds. A short computation verifies that

$$
\left.j^{5}(\varphi)^{*}(P\rfloor \Omega_{L}\right)=0
$$

where $P \in T J^{5}(Y)$ and is $T_{\pi_{Y, J^{5}(Y)}}$-vertical. For any $W \in T J^{5}(Y)$, there exists vector field $V$, such that

$$
W=j^{5}(V)+P
$$

So, by (2.8)-(2.10), if $\varphi$ is an extremum of $\left.S, j^{5}(\varphi)^{*}(W\rfloor \Omega_{L}\right)$ mush vanish for any vector field $W \in T J^{5}(Y)$, thus, we get the Euler-lagrange equation

$$
\left.j^{5}(\varphi) *(W\rfloor \Omega_{L}\right)=0
$$

for any vector field $W \in T J^{5}(Y)$. In the following part, we consider the multisymplectic form formula and a corollary of the multisymplectic form formula. About the multisymplectic form formula for first order field theories, please refer to [11].

Theorem 2.3 let $\eta_{Y}^{\lambda}$ and $\xi_{Y}^{\lambda}$ demote two one-parameter symmetry groups of equation (2.11) and the corresponding vector fields are $V$ and $W$. Then we have the multisymplectic form formula

$$
\left.\left.\int_{\partial U} j^{5}(\varphi)^{*}\left(j^{5}(V)\right\rfloor j^{5}(W)\right\rfloor \Omega_{L}\right)=0 .
$$

Proof: Since $j^{5}[W, V]=\left[j^{5}(W), j^{5}(V)\right]$, from (2.11) we have

$$
\begin{aligned}
0 & \left.=\int_{U} j^{5}(\varphi)^{*}\left(j^{5}[W, V]\right] \Omega_{L}\right) \\
& \left.=\int_{U} j^{5}(\varphi)^{*}\left(\left[j^{5}(W), j^{5}(V)\right]\right\rfloor \Omega_{L}\right) \\
& \left.\left.=\int_{U} j^{5}(\varphi)^{*}\left(£_{j^{5}(W)}\left(j^{5}(V)\right\rfloor \Omega_{L}\right)-j^{5}(V)\right\rfloor{j^{5}(W)} \Omega_{L}\right) .
\end{aligned}
$$

Because $\eta_{Y}^{\lambda}$ and $\xi_{Y}^{\lambda}$ are two one-parameter symmetry groups of equation (2.11), so for any vector field $Q \in T J^{5}(Y)$, we have

$$
\left.\left.\left.\frac{d}{d \lambda}\right|_{\lambda=0} j^{5}\left(\eta_{Y}^{\lambda} \circ \varphi \circ\left(\eta_{X}^{\lambda}\right)^{-1}\right)^{*}(Q\rfloor \Omega_{L}\right)=j^{5}(\varphi)^{*} £_{j^{5}(V)}(Q\rfloor \Omega_{L}\right)=0,
$$




$$
\left.\left.\left.\frac{d}{d \lambda}\right|_{\lambda=0} j^{5}\left(\xi_{Y}^{\lambda} \circ \varphi \circ\left(\xi_{X}^{\lambda}\right)^{-1}\right)^{*}(Q\rfloor \Omega_{L}\right)=j^{5}(\varphi)^{*} £_{j^{5}(W)}(Q\rfloor \Omega_{L}\right)=0 .
$$

Thus (2.13) becomes

$$
\begin{aligned}
0 & \left.=-\int_{U} j^{5}(\varphi)^{*}\left(j^{5}(V)\right\rfloor £_{j^{5}(W)} \Omega_{L}\right) \\
& \left.\left.=-\int_{U} j^{5}(\varphi)^{*}\left(j^{5}(V)\right\rfloor d\left(j^{5}(W)\right\rfloor \Omega_{L}\right)\right) \\
& \left.=\int_{U} j^{5}(\varphi)^{*}\left(j^{5}(V)\right\rfloor d\left(£_{j^{5}(W)} \theta_{L}\right)\right) .
\end{aligned}
$$

$\left.\left.j^{5}(V)\right\rfloor j^{5}(W)\right\rfloor \Omega_{L}$ can be written as

$$
\begin{aligned}
\left.\left.j^{5}(V)\right\rfloor j^{5}(W)\right\rfloor \Omega_{L} & \left.\left.\left.=j^{5}(V)\right\rfloor d\left(j^{5}(W)\right\rfloor \theta_{L}\right)-j^{5}(V)\right\rfloor £_{j^{5}(W)} \theta_{L} \\
& \left.\left.\left.\left.=£_{j^{5}(V)}\left(j^{5}(W)\right\rfloor \theta_{L}\right)-j^{5}(V)\right\rfloor \sum_{j^{5}(W)} \theta_{L}-d\left(j^{5}(V)\right\rfloor j^{5}(W)\right\rfloor \theta_{L}\right) .
\end{aligned}
$$

So from stokes' formula we can obtain that

$$
\begin{aligned}
& \left.\left.\int_{\partial U} j^{5}(\varphi)^{*}\left(j^{5}(V)\right\rfloor j^{5}(W)\right\rfloor \Omega_{L}\right) \\
= & \left.\left.\left.\left.\int_{\partial U} j^{5}(\varphi)^{*}\left(£_{j^{5}(V)}\left(j^{5}(W)\right\rfloor \theta_{L}\right)-j^{5}(V)\right\rfloor £_{j^{5}(W)} \theta_{L}-d\left(j^{5}(V)\right\rfloor j^{5}(W)\right\rfloor \theta_{L}\right)\right) \\
= & \left.\left.\int_{U} j^{5}(\varphi)^{*} d\left(£_{j^{5}(V)}\left(j^{5}(W)\right\rfloor \theta_{L}\right)-j^{5}(V)\right\rfloor j_{j^{5}(W)} \theta_{L}\right) \\
= & \left.\left.\int_{U} j^{5}(\varphi)^{*}\left(£_{j^{5}(V)} \sum_{j^{5}(W)} \theta_{L}+£_{j^{5}(V)}\left(j^{5}(W)\right\rfloor \Omega_{L}\right)-d\left(j^{5}(V)\right\rfloor £_{j^{5}(W)} \theta_{L}\right)\right) .
\end{aligned}
$$

By

$$
\left.\left.£_{j^{5}(V)} £_{j^{5}(W)} \theta_{L}=j^{5}(V)\right\rfloor d\left(£_{j^{5}(W)} \theta_{L}\right)+d\left(j^{5}(V)\right\rfloor £_{j^{5}(W)} \theta_{L}\right),
$$

we have

$$
\begin{aligned}
& \left.\left.\int_{\partial U} j^{5}(\varphi)^{*}\left(j^{5}(V)\right\rfloor j^{5}(W)\right\rfloor \Omega_{L}\right) \\
= & \left.\left.\int_{U} j^{5}(\varphi)^{*}\left(j^{5}(V)\right\rfloor d\left(£_{j^{5}(W)} \theta_{L}\right)+£_{j^{5}(V)}\left(j^{5}(W)\right\rfloor \Omega_{L}\right)\right) .
\end{aligned}
$$

Hence, by (2.14) and (2.16), we obtain

$$
\left.\left.\int_{\partial U} j^{5}(\varphi)^{*}\left(j^{5}(V)\right\rfloor j^{5}(W)\right\rfloor \Omega_{L}\right)=0 .
$$

Although the covariant Legendre transformation ( or complete Legendre transformation ) which transform time and space variable simultaneously are not necessarily unique, for this fixed Cartan form $\theta_{L}$, we can construct corresponding covariant Lengendre transformation of Lagrangian density $L$. Let $v=\varphi_{x}, u=\varphi_{x x}, w=\varphi_{x y}, p=\varphi_{x t}$, taking the covariant Legendre transform of Lagrangian density $L$ :

$$
\begin{aligned}
& p^{x}=-2 \varphi_{x x t}-6 \varphi_{x x} \varphi_{x x x}-\sigma \varphi_{x y y}-\varphi_{x x x x x} \\
& p^{x x}=\varphi_{x t}+3 \varphi_{x x}^{2}+\varphi_{x x x x}, \quad p^{x t}=\varphi_{x x} \\
& p^{x y}=\sigma \varphi_{x y}, \quad p^{x x x}=-\varphi_{x x x}
\end{aligned}
$$

According to the covariant De Donder-Weyl Hamilton function theories [18] and the multisymplectic concept introduced by Bridges [3], KP equation can be reformulated as a system of ten first-order partial differential equations which can be written in the form:

$$
\begin{array}{r}
M \mathbb{Z}_{t}+K \mathbb{Z}_{x}+L \mathbb{Z}_{y}=\nabla S(\mathbb{Z}), \\
\mathbb{Z}=\left(\varphi, v, u, w, p, p^{x}, p^{x x}, p^{x y}, p^{x t}, p^{x x x}\right)^{T} \in \mathbb{R}^{10},
\end{array}
$$


where

$$
\begin{aligned}
& M=\left(\begin{array}{cccccccccc}
0 & 0 & 0 & 0 & 0 & 0 & 0 & 0 & 0 & 0 \\
0 & 0 & 0 & 0 & 0 & 0 & 0 & 0 & 1 & 0 \\
0 & 0 & 0 & 0 & 0 & 0 & 0 & 0 & 0 & 0 \\
0 & 0 & 0 & 0 & 0 & 0 & 0 & 0 & 0 & 0 \\
0 & 0 & 0 & 0 & 0 & 0 & 0 & 0 & 0 & 0 \\
0 & 0 & 0 & 0 & 0 & 0 & 0 & 0 & 0 & 0 \\
0 & 0 & 0 & 0 & 0 & 0 & 0 & 0 & 0 & 0 \\
0 & 0 & 0 & 0 & 0 & 0 & 0 & 0 & 0 & 0 \\
0 & -1 & 0 & 0 & 0 & 0 & 0 & 0 & 0 & 0 \\
0 & 0 & 0 & 0 & 0 & 0 & 0 & 0 & 0 & 0
\end{array}\right) \\
& K=\left(\begin{array}{cccccccccc}
0 & 0 & 0 & 0 & 0 & 1 & 0 & 0 & 0 & 0 \\
0 & 0 & 0 & 0 & 0 & 0 & 1 & 0 & 0 & 0 \\
0 & 0 & 0 & 0 & 0 & 0 & 0 & 0 & 0 & 1 \\
0 & 0 & 0 & 0 & 0 & 0 & 0 & 0 & 0 & 0 \\
0 & 0 & 0 & 0 & 0 & 0 & 0 & 0 & 0 & 0 \\
-1 & 0 & 0 & 0 & 0 & 0 & 0 & 0 & 0 & 0 \\
0 & -1 & 0 & 0 & 0 & 0 & 0 & 0 & 0 & 0 \\
0 & 0 & 0 & 0 & 0 & 0 & 0 & 0 & 0 & 0 \\
0 & 0 & 0 & 0 & 0 & 0 & 0 & 0 & 0 & 0 \\
0 & 0 & -1 & 0 & 0 & 0 & 0 & 0 & 0 & 0
\end{array}\right) \\
& L=\left(\begin{array}{cccccccccc}
0 & 0 & 0 & 0 & 0 & 0 & 0 & 0 & 0 & 0 \\
0 & 0 & 0 & 0 & 0 & 0 & 0 & 1 & 0 & 0 \\
0 & 0 & 0 & 0 & 0 & 0 & 0 & 0 & 0 & 0 \\
0 & 0 & 0 & 0 & 0 & 0 & 0 & 0 & 0 & 0 \\
0 & 0 & 0 & 0 & 0 & 0 & 0 & 0 & 0 & 0 \\
0 & 0 & 0 & 0 & 0 & 0 & 0 & 0 & 0 & 0 \\
0 & 0 & 0 & 0 & 0 & 0 & 0 & 0 & 0 & 0 \\
0 & -1 & 0 & 0 & 0 & 0 & 0 & 0 & 0 & 0 \\
0 & 0 & 0 & 0 & 0 & 0 & 0 & 0 & 0 & 0 \\
0 & 0 & 0 & 0 & 0 & 0 & 0 & 0 & 0 & 0
\end{array}\right) \\
& S(\mathbb{Z})=u p+\frac{1}{2}\left(p^{x x x}\right)^{2}+\frac{\sigma}{2} w^{2}+u^{3}-p^{x} v-p^{x x} u-p^{x t} p-p^{x y} w .
\end{aligned}
$$

$\nabla S$ is the gradient of $S$ with respect to the standard inner product on $\mathbb{R}^{10}$. The system (2.21) is a Hamiltonian formulation of the KP equation on a multisymplectic structure. Although this formulation is not the best, we give a constructive method to get multisymplectic form which proposed by Bridges [3]. It should be pay attention that (2.21) is different with the multisymplectic form of $\mathrm{KP}$ equation in [4]. For equation (2.21), there is a conservation law

$$
\partial_{t}(d \mathbb{Z} \wedge M d \mathbb{Z})+\partial_{x}(d \mathbb{Z} \wedge K d \mathbb{Z})+\partial_{y}(d \mathbb{Z} \wedge L d \mathbb{Z})=0 .
$$


Substituting $M, K, L$ into (2.22) leads to

$$
\begin{aligned}
& \frac{\partial}{\partial t}\left(d \varphi_{x} \wedge d \varphi_{x x}\right)+\frac{\partial}{\partial x}\left(d \varphi \wedge\left(-6 \varphi_{x x x} d \varphi_{x x}-6 \varphi_{x x} d \varphi_{x x x}-\sigma d \varphi_{x y y}-2 d \varphi_{x x t}-d \varphi_{x x x x x}\right)\right. \\
& \left.+d \varphi_{x} \wedge\left(6 \varphi_{x x} d \varphi_{x x}+d \varphi_{x t}+d \varphi_{x x x x}\right)+d \varphi_{x x} \wedge d\left(-\varphi_{x x x}\right)\right)+\frac{\partial}{\partial y}\left(d \varphi_{x} \wedge d\left(\sigma \varphi_{x y}\right)\right)=0 .
\end{aligned}
$$

This multisymplectic conservation law (2.22) consistent with our theorem 2.3. We can regard the conservation law (2.23) as a corollary of the theorem 2.3. Let $V, W$ be $\pi_{X Y}$-vertical and have the expressions $V^{\varphi} \frac{\partial}{\partial \varphi}, W^{\varphi} \frac{\partial}{\partial \varphi}$. Thus the corresponding $j^{5}(V)$ and $j^{5}(W)$ have the coordinate expressions $\left(V^{\varphi}, V_{x}^{\varphi}, V_{y}^{\varphi}, V_{t}^{\varphi}, V_{x x}^{\varphi}, V_{y y}^{\varphi}, V_{t t}^{\varphi}, V_{x t}^{\varphi}, V_{x y}^{\varphi}, V_{y t}^{\varphi}, V_{x x x}^{\varphi}\right.$, $V_{x t x}^{\varphi}, V_{x x y}^{\varphi}, V_{x t t}^{\varphi}, V_{x y y}^{\varphi}, V_{t t t}^{\varphi}, V_{y y y}^{\varphi}, V_{t y y}^{\varphi}, V_{t t y}^{\varphi}, V_{x x x x}^{\varphi}, V_{x x y y}^{\varphi}, V_{x x t t}^{\varphi}, V_{x x x y}^{\varphi}, V_{x x x t}^{\varphi}, V_{x y y y}^{\varphi}, V_{x t t t}^{\varphi}, V_{t t t t}^{\varphi}$, $V_{y y y y}^{\varphi}, V_{t y y y}^{\varphi}, V_{t t y y}^{\varphi}, V_{t t t y}^{\varphi}, V_{x x x x x}^{\varphi}, V_{x x x x y}^{\varphi}, V_{x x x x t}^{\varphi}, V_{x x x y y}^{\varphi}, V_{x x x t t}^{\varphi}, V_{x x y y y}^{\varphi}, V_{x x t t t}^{\varphi}, V_{x y y y y}^{\varphi}, V_{x t t t t}^{\varphi}$, $\left.V_{y y y y y}^{\varphi}, V_{t t t t t}^{\varphi}, V_{y y y y t}^{\varphi}, V_{y y y t t}^{\varphi}, V_{y y t t t}^{\varphi}, V_{y t t t t}^{\varphi}\right)$ and $\left(W^{\varphi}, W_{x}^{\varphi}, W_{y}^{\varphi}, W_{t}^{\varphi}, W_{x x}^{\varphi}, W_{y y}^{\varphi}, W_{t t}^{\varphi}, W_{x t}^{\varphi}, W_{x y}^{\varphi}\right.$, $W_{y t}^{\varphi}, W_{x x x}^{\varphi}, W_{x t x}^{\varphi}, W_{x x y}^{\varphi}, W_{x t t}^{\varphi}, W_{x y y}^{\varphi}, W_{t t t}^{\varphi}, W_{y y y}^{\varphi}, W_{t y y}^{\varphi}, W_{t t y}^{\varphi}, W_{x x x x}^{\varphi}, W_{x x y y}^{\varphi}, W_{x x t t}^{\varphi}, W_{x x x y}^{\varphi}$, $W_{x x x t}^{\varphi}, W_{x y y y}^{\varphi}, W_{x t t t}^{\varphi}, W_{t t t t}^{\varphi}, W_{y y y y}^{\varphi}, W_{t y y y}^{\varphi}, W_{t t y y}^{\varphi}, W_{t t t y}^{\varphi}, W_{x x x x x}^{\varphi}, W_{x x x x y}^{\varphi}, W_{x x x x t}^{\varphi}, W_{x x x y y}^{\varphi}$, $\left.W_{x x x t t}^{\varphi}, W_{x x y y y}^{\varphi}, W_{x x t t t}^{\varphi}, W_{x y y y y}^{\varphi}, W_{x t t t t}^{\varphi}, W_{y y y y y}^{\varphi}, W_{t t t t t}^{\varphi}, W_{y y y y t}^{\varphi}, W_{y y y t t}^{\varphi}, W_{y y t t t}^{\varphi}, W_{y t t t t}^{\varphi}\right)$.

We can compute

$$
\begin{aligned}
& \left.\left.j^{5}(\varphi)^{*}\left(j^{5}(V)\right\rfloor j^{5}(W)\right\rfloor \Omega_{L}\right)=\left(W_{x x}^{\varphi} V_{x}^{\varphi}-W_{x}^{\varphi} V_{x x}^{\varphi}\right) d x \wedge d y \\
& +\left(\sigma W_{x}^{\varphi} V_{x y}^{\varphi}-\sigma W_{x y}^{\varphi} V_{x}^{\varphi}\right) d x \wedge d t+\left[W_{x x}^{\varphi} V_{x x x}^{\varphi}-W_{x x x}^{\varphi} V_{x x}^{\varphi}\right. \\
& -W_{x}^{\varphi}\left(V_{x t}^{\varphi}+6 \varphi_{x x} V_{x x}^{\varphi}+V_{x x x x}^{\varphi}\right)+V_{x}^{\varphi}\left(W_{x t}^{\varphi}+6 \varphi_{x x} W_{x x}^{\varphi}+W_{x x x x}^{\varphi}\right) \\
& +V^{\varphi}\left(-2 W_{x x t}^{\varphi}-6 \varphi_{x x} W_{x x x}^{\varphi}-6 \varphi_{x x x} W_{x x}^{\varphi}-\sigma W_{x y y}^{\varphi}-W_{x x x x x}^{\varphi}\right) \\
& \left.+W^{\varphi}\left(2 V_{x x t}^{\varphi}+6 \varphi_{x x} V_{x x x}^{\varphi}+6 \varphi_{x x x} V_{x x}^{\varphi}+\sigma V_{x y y}^{\varphi}+V_{x x x x x}^{\varphi}\right)\right] d y \wedge d t .
\end{aligned}
$$

By the Stokes' formula, we can obtain

$$
\begin{aligned}
& \int_{U} \frac{\partial}{\partial t}\left(W_{x x}^{\varphi} V_{x}^{\varphi}-W_{x}^{\varphi} V_{x x}^{\varphi}\right) d x \wedge d y \wedge d t-\frac{\partial}{\partial y}\left(\sigma W_{x}^{\varphi} V_{x y}^{\varphi}-\sigma W_{x y}^{\varphi} V_{x}^{\varphi}\right) d x \wedge d y \wedge d t \\
& +\frac{\partial}{\partial x}\left[W_{x x}^{\varphi} V_{x x x}^{\varphi}-W_{x x x}^{\varphi} V_{x x}^{\varphi}-W_{x}^{\varphi}\left(V_{x t}^{\varphi}+6 \varphi_{x x} V_{x x}^{\varphi}+V_{x x x x}^{\varphi}\right)+V_{x}^{\varphi}\left(W_{x t}^{\varphi}+6 \varphi_{x x} W_{x x}^{\varphi}\right.\right. \\
& \left.+W_{x x x x}^{\varphi}\right)+V^{\varphi}\left(-2 W_{x x t}^{\varphi}-6 \varphi_{x x} W_{x x x}^{\varphi}-6 \varphi_{x x x} W_{x x}^{\varphi}-\sigma W_{x y y}^{\varphi}-W_{x x x x x}^{\varphi}\right) \\
& \left.+W^{\varphi}\left(2 V_{x x t}^{\varphi}+6 \varphi_{x x} V_{x x x}^{\varphi}+6 \varphi_{x x x} V_{x x}^{\varphi}+\sigma V_{x y y}^{\varphi}+V_{x x x x x}^{\varphi}\right)\right] d x \wedge d y \wedge d t=0 .
\end{aligned}
$$

Since $U$ is arbitrary, we get

$$
\begin{aligned}
& \frac{\partial}{\partial t}\left(W_{x x}^{\varphi} V_{x}^{\varphi}-W_{x}^{\varphi} V_{x x}^{\varphi}\right)-\frac{\partial}{\partial y}\left(\sigma W_{x}^{\varphi} V_{x y}^{\varphi}-\sigma W_{x y}^{\varphi} V_{x}^{\varphi}\right)+\frac{\partial}{\partial x}\left[W_{x x}^{\varphi} V_{x x x}^{\varphi}-\right. \\
& W_{x x x}^{\varphi} V_{x x}^{\varphi}-W_{x}^{\varphi}\left(V_{x t}^{\varphi}+6 \varphi_{x x} V_{x x}^{\varphi}+V_{x x x x}^{\varphi}\right)+V_{x}^{\varphi}\left(W_{x t}^{\varphi}+6 \varphi_{x x} W_{x x}^{\varphi}+W_{x x x x}^{\varphi}\right) \\
& +V^{\varphi}\left(-2 W_{x x t}^{\varphi}-6 \varphi_{x x} W_{x x x}^{\varphi}-6 \varphi_{x x x} W_{x x}^{\varphi}-\sigma W_{x y y}^{\varphi}-W_{x x x x x}^{\varphi}\right) \\
& \left.+W^{\varphi}\left(2 V_{x x t}^{\varphi}+6 \varphi_{x x} V_{x x x}^{\varphi}+6 \varphi_{x x x} V_{x x}^{\varphi}+\sigma V_{x y y}^{\varphi}+V_{x x x x x}^{\varphi}\right)\right]=0
\end{aligned}
$$

If we let

$$
M^{t}=\left(\begin{array}{cccccccccc}
0 & 0 & 0 & 0 & 0 & 0 & 0 & 0 & 0 & 0 \\
0 & 0 & 1 & 0 & 0 & 0 & 0 & 0 & 0 & 0 \\
0 & -1 & 0 & 0 & 0 & 0 & 0 & 0 & 0 & 0 \\
0 & 0 & 0 & 0 & 0 & 0 & 0 & 0 & 0 & 0 \\
0 & 0 & 0 & 0 & 0 & 0 & 0 & 0 & 0 & 0 \\
0 & 0 & 0 & 0 & 0 & 0 & 0 & 0 & 0 & 0 \\
0 & 0 & 0 & 0 & 0 & 0 & 0 & 0 & 0 & 0 \\
0 & 0 & 0 & 0 & 0 & 0 & 0 & 0 & 0 & 0 \\
0 & 0 & 0 & 0 & 0 & 0 & 0 & 0 & 0 & 0 \\
0 & 0 & 0 & 0 & 0 & 0 & 0 & 0 & 0 & 0
\end{array}\right)
$$




$$
M^{x}=\left(\begin{array}{cccccccccc}
0 & 0 & -6 \varphi_{x x x} & 0 & 0 & -6 \varphi_{x x} & -\sigma & -2 & 0 & -1 \\
0 & 0 & 6 \varphi_{x x} & 0 & 1 & 0 & 0 & 0 & 1 & 0 \\
6 \varphi_{x x x} & -6 \varphi_{x x} & 0 & 0 & 0 & -1 & 0 & 0 & 0 & 0 \\
0 & 0 & 0 & 0 & 0 & 0 & 0 & 0 & 0 & 0 \\
0 & -1 & 0 & 0 & 0 & 0 & 0 & 0 & 0 & 0 \\
6 \varphi_{x x} & 0 & 1 & 0 & 0 & 0 & 0 & 0 & 0 & 0 \\
\sigma & 0 & 0 & 0 & 0 & 0 & 0 & 0 & 0 & 0 \\
2 & 0 & 0 & 0 & 0 & 0 & 0 & 0 & 0 & 0 \\
0 & -1 & 0 & 0 & 0 & 0 & 0 & 0 & 0 & 0 \\
1 & 0 & 0 & 0 & 0 & 0 & 0 & 0 & 0 & 0
\end{array}\right)
$$

$$
\begin{gathered}
M^{y}=\left(\begin{array}{cccccccccc}
0 & 0 & 0 & 0 & 0 & 0 & 0 & 0 & 0 & 0 \\
0 & 0 & 0 & \sigma & 0 & 0 & 0 & 0 & 0 & 0 \\
0 & 0 & 0 & 0 & 0 & 0 & 0 & 0 & 0 & 0 \\
0 & -\sigma & 0 & 0 & 0 & 0 & 0 & 0 & 0 & 0 \\
0 & 0 & 0 & 0 & 0 & 0 & 0 & 0 & 0 & 0 \\
0 & 0 & 0 & 0 & 0 & 0 & 0 & 0 & 0 & 0 \\
0 & 0 & 0 & 0 & 0 & 0 & 0 & 0 & 0 & 0 \\
0 & 0 & 0 & 0 & 0 & 0 & 0 & 0 & 0 & 0 \\
0 & 0 & 0 & 0 & 0 & 0 & 0 & 0 & 0 & 0 \\
0 & 0 & 0 & 0 & 0 & 0 & 0 & 0 & 0 & 0
\end{array}\right) \\
M^{t}(x, y)=x^{T} M^{t} y, M^{x}(x, y)=x^{T} M^{x} y, M^{y}(x, y)=x^{T} M^{y} y \\
j^{5}(V)=\left(V^{\varphi}, V_{x}^{\varphi}, V_{x x}^{\varphi}, V_{x y}^{\varphi}, V_{x t}^{\varphi}, V_{x x x}^{\varphi}, V_{x y y}^{\varphi}, V_{x x t}^{\varphi}, V_{x x x x}^{\varphi}, V_{x x x x x}^{\varphi}\right) \\
j^{5}(W)=\left(W^{\varphi}, W_{x}^{\varphi}, W_{x x}^{\varphi}, W_{x y}^{\varphi}, W_{x t}^{\varphi}, W_{x x x}^{\varphi}, W_{x y y}^{\varphi}, W_{x x t}^{\varphi}, W_{x x x x}^{\varphi}, W_{x x x x x}^{\varphi}\right)
\end{gathered}
$$

and set

the others coordinates vanished. Then conservation law (2.24) can be written as

$$
\frac{\partial}{\partial t} M^{t}\left(j^{5}(V), j^{5}(W)\right)+\frac{\partial}{\partial x} M^{x}\left(j^{5}(V), j^{5}(W)\right)+\frac{\partial}{\partial y} M^{y}\left(j^{5}(V), j^{5}(W)\right)=0
$$

Also since the translation invariance of the $\mathrm{KP}$ equation, we choose $V=W=\varphi$ and take it into (2.26), conservation law (2.26) becomes:

$$
\begin{aligned}
& \frac{\partial}{\partial t}\left(d \varphi_{x} \wedge d \varphi_{x x}\right)+\frac{\partial}{\partial x}\left(d \varphi \wedge\left(-6 \varphi_{x x x} d \varphi_{x x}-6 \varphi_{x x} d \varphi_{x x x}-\sigma d \varphi_{x y y}-2 d \varphi_{x x t}-d \varphi_{x x x x x}\right)\right. \\
& \left.+d \varphi_{x} \wedge\left(6 \varphi_{x x} d \varphi_{x x}+d \varphi_{x t}+d \varphi_{x x x x}\right)+d \varphi_{x x} \wedge d\left(-\varphi_{x x x}\right)\right)+\frac{\partial}{\partial y}\left(d \varphi_{x} \wedge d\left(\sigma \varphi_{x y}\right)\right)=0 .
\end{aligned}
$$

i.e. the conservation law (2.23).

In the numerical study, the multisymplectic conservation law can be used to design multisymplectic numerical schemes, i.e., numerical schemes which can preserve the multisymplectic conservation law. 


\section{$3 \quad$ Multisymplectic Preissman Scheme for the KP Equation}

In this section, we consider the multisymplectic Preissman scheme for the KP equation. The equation (2.21) can be reformulated as

$$
\left\{\begin{array}{l}
\frac{\partial p^{x}}{\partial x}=0, \\
\frac{\partial p^{x x}}{\partial x}+\frac{\partial p^{x y}}{\partial y}+\frac{\partial p^{x t}}{\partial t}=-p^{x}, \\
\frac{\partial p^{x x x}}{\partial x}=p+3 u^{2}-p^{x x}, \\
\sigma w-p^{x y}=0, \\
u-p^{x t}=0, \\
\frac{\partial \varphi}{\partial x}=v \\
\frac{\partial v}{\partial x}=u \\
\frac{\partial v}{\partial y}=w \\
\frac{\partial v}{\partial t}=p \\
\frac{\partial u}{\partial x}=p^{x x x}
\end{array}\right.
$$

For convenience, we assumed that the spacing of the grid points in the $x, y, t$ directions is uniform respectively. We apply the implicit midpoint discretization in time and in space to (3.1), and obtain that:

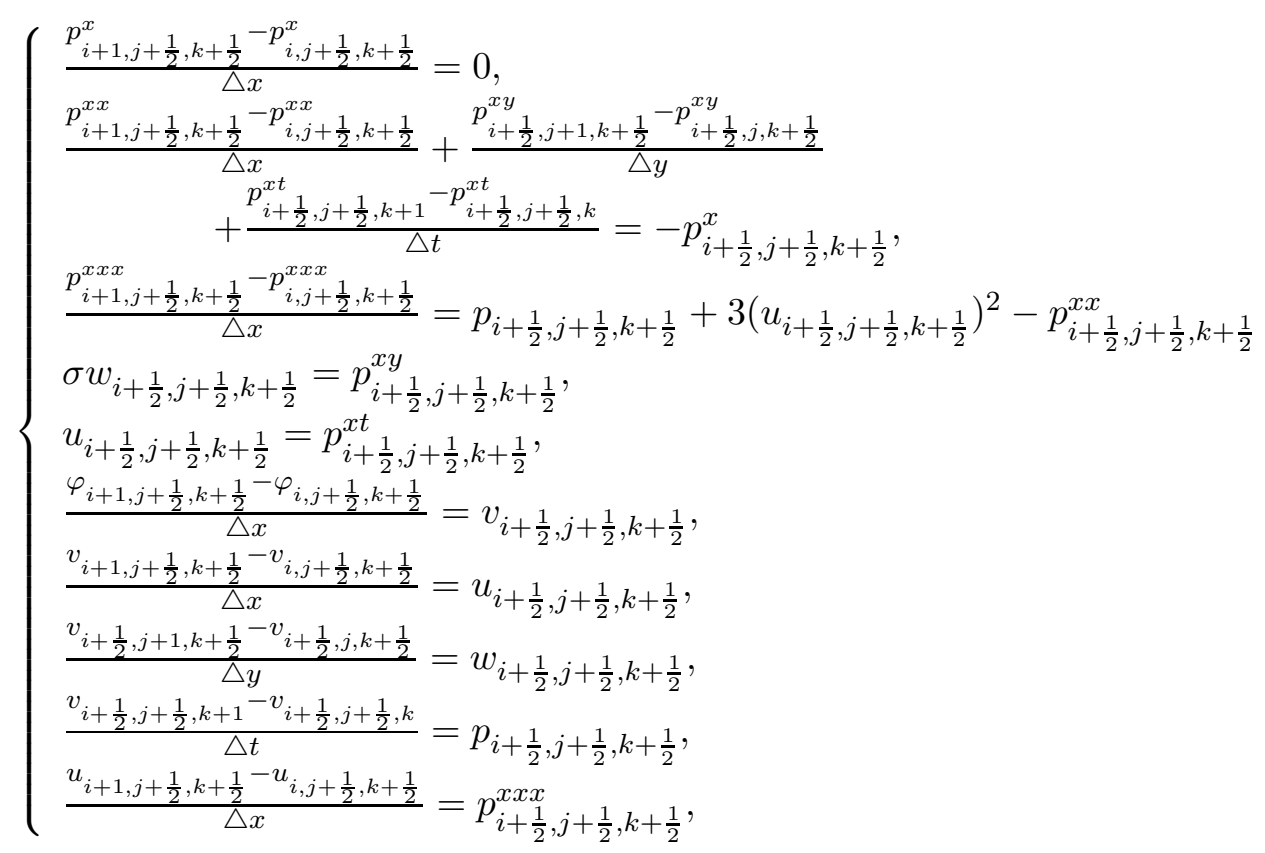

Where $\triangle x$ is the $x$-direction step, $\triangle y$ is the $y$-direction step, $\triangle t$ is the time step and $u_{i+\frac{1}{2}, j+\frac{1}{2}, k+\frac{1}{2}}=u\left(i \triangle x+\frac{\triangle x}{2}, j \triangle y+\frac{\triangle y}{2}, k \triangle t+\frac{\Delta t}{2}\right)$, the others is similar.

In fact, the discretization result lead to the pressman scheme

$$
\begin{aligned}
& \frac{1}{\triangle t} M\left(\mathbb{Z}_{i+\frac{1}{2}, j+\frac{1}{2}, k+1}-\mathbb{Z}_{i+\frac{1}{2}, j+\frac{1}{2}, k}\right)+\frac{1}{\triangle x} K\left(\mathbb{Z}_{i+1, j+\frac{1}{2}, k+\frac{1}{2}}-\mathbb{Z}_{i, j+\frac{1}{2}, k+\frac{1}{2}}\right) \\
& +\frac{1}{\triangle y} L\left(\mathbb{Z}_{i+\frac{1}{2}, j+1, k+\frac{1}{2}}-\mathbb{Z}_{i+\frac{1}{2}, j, k+\frac{1}{2}}\right)=\nabla S\left(\mathbb{Z}_{i+\frac{1}{2}, j+\frac{1}{2}, k+\frac{1}{2}}\right)
\end{aligned}
$$


The (3.3) preserve the discrete multisymplectic conservation law:

$$
\begin{aligned}
& \frac{d v_{i+\frac{1}{2}, j+\frac{1}{2}, k+1} \wedge d p_{i+\frac{1}{2}, j+\frac{1}{2}, k+1}^{x t}-d v_{i+\frac{1}{2}, j+\frac{1}{2}, k} \wedge d p_{i+\frac{1}{2}, j+\frac{1}{2}, k}^{x t}}{\triangle t}+ \\
& \frac{\frac{d v_{i+\frac{1}{2}, j+1, k+\frac{1}{2}} \wedge d p_{i+\frac{1}{2}, j+1, k+\frac{1}{2}}^{x y}-d v_{i+\frac{1}{2}, j, k+\frac{1}{2}} \wedge d p_{i+\frac{1}{2}, j, k+\frac{1}{2}}^{x y}}{\triangle y}+}{\frac{d \varphi_{i+1, j+\frac{1}{2}, k+\frac{1}{2}} \wedge d p_{i+1, j+\frac{1}{2}, k+\frac{1}{2}}^{x}-d \varphi_{i, j+\frac{1}{2}, k+\frac{1}{2}} \wedge d p_{i, j+\frac{1}{2}, k+\frac{1}{2}}^{x}}{\triangle x}+} \\
& \frac{d v_{i+1, j+\frac{1}{2}, k+\frac{1}{2}} \wedge d p_{i+1, j+\frac{1}{2}, k+\frac{1}{2}}^{x x}-d v_{i, j+\frac{1}{2}, k+\frac{1}{2}} \wedge d p_{i, j+\frac{1}{2}, k+\frac{1}{2}}^{x x}}{\triangle x}+ \\
& \frac{d u_{i+1, j+\frac{1}{2}, k+\frac{1}{2}} \wedge d p_{i+1, j+\frac{1}{2}, k+\frac{1}{2}}^{x x x}-d u_{i, j+\frac{1}{2}, k+\frac{1}{2}} \wedge d p_{i, j+\frac{1}{2}, k+\frac{1}{2}}^{x x x}}{\triangle x}=0
\end{aligned}
$$

Although the Preissman scheme (3.3) is multisymplectic, it took too efforts to realize. Hence we elimate the auxiliary variables $\varphi, v, w, p, p^{x}, p^{x x}, p^{x y}, p^{x t}, p^{x x x}$ by a trivial computation and obtain the following multisymplectic forty-five points scheme:

$$
\begin{aligned}
& \frac{1}{2 \triangle x \triangle t} \delta_{y}^{2} \Delta_{t}^{0}\left\{u_{i+2, j}^{k}+2 u_{i+1, j}^{k}-2 u_{i-1, j}^{k}+u_{i-2, j}^{k}\right\} \\
& \quad+\frac{1}{\triangle x^{4}}\left\{\delta_{y}^{2} \delta_{t}^{2}\left(u_{i+2, j}^{k}-4 u_{i+1, j}^{k}+6 u_{i, j}^{k}-4 u_{i-1, j}^{k}+u_{i-2, j}^{k}\right)\right\} \\
& \quad+\frac{\sigma}{4 \triangle y^{2}}\left\{\delta_{t}^{2} \Delta_{y}^{2}\left(u_{i+2, j}^{k}+4 u_{i+1, j}^{k}+6 u_{i, j}^{k}+4 u_{i-1, j}^{k}+u_{i, j}^{k}\right)\right\} \\
& \quad+\frac{2}{\triangle x^{2}}\left(\bar{\delta} f_{i, j+\frac{1}{2}}^{k+\frac{1}{2}}+\bar{\delta} f_{i, j-\frac{1}{2}}^{k+\frac{1}{2}}+\bar{\delta} f_{i, j+\frac{1}{2}}^{k-\frac{1}{2}}+\bar{\delta} f_{i, j-\frac{1}{2}}^{k-\frac{1}{2}}\right)=0
\end{aligned}
$$

where we denote $u_{i, j}^{k}=u(i \triangle x, j \triangle y, k \triangle t), f_{i, j}^{k}=3(u(i \triangle x, j \triangle y, k \triangle t))^{2}$ and

$$
\left\{\begin{array}{l}
\Delta_{t}^{0} u_{i, j}^{k}=u_{i, j}^{k+1}-u_{i, j}^{k-1}, \delta_{y}^{2} u_{i, j}^{k}=u_{i, j+1}^{k}+2 u_{i, j}^{k}+u_{i, j-1}^{k}, \\
\delta_{t}^{2} u_{i, j}^{k}=u_{i, j}^{k+1}+2 u_{i, j}^{k}+u_{i, j}^{k-1}, \Delta_{y}^{2} u_{i, j}^{k}=u_{i, j+1}^{k}-2 u_{i, j}^{k}+u_{i, j-1}^{k}, \\
\bar{\delta} f_{i, j}^{k}=f_{i+\frac{3}{2}, j}^{k}-f_{i+\frac{1}{2}, j}^{k}-f_{i-\frac{1}{2}, j}^{k}+f_{i-\frac{3}{2}, j}^{k}
\end{array}\right.
$$

\section{Some Numerical Results on Soliton and Solitary Waves}

In this section, we test the forty-five points scheme on soliton and solitary waves over long time intervals.

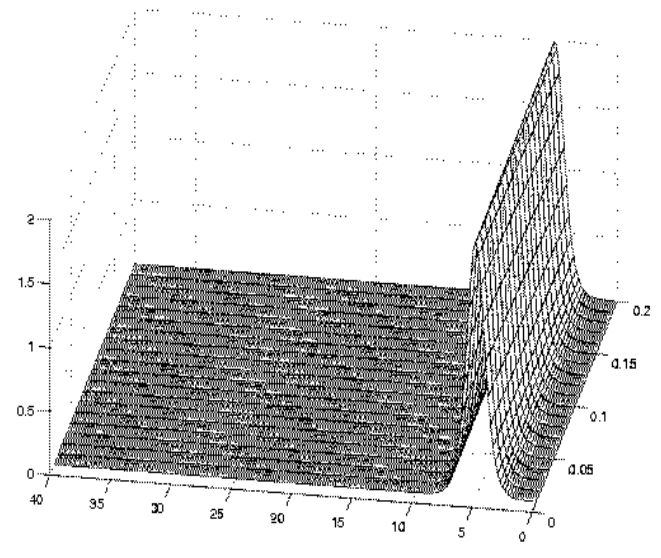

Fig.1: one soliton at $\mathrm{t}=0$,

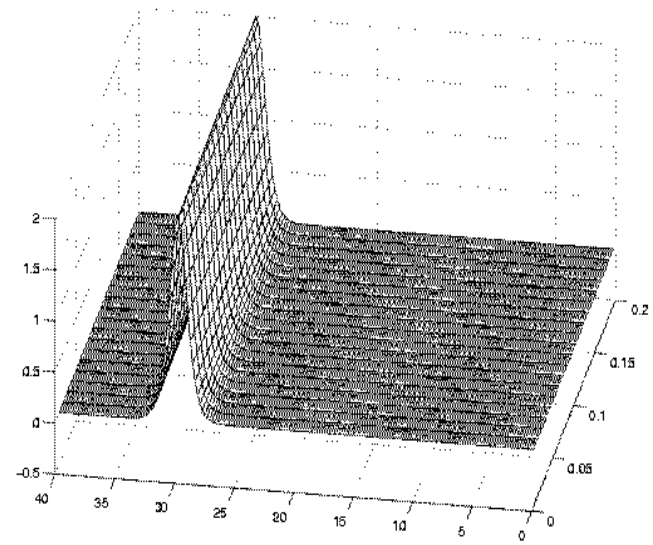

Fig.2: one soliton at $\mathrm{t}=10$. 
At first, one line soliton of KPI equation is considered. We choose small interval on $y$-direction just for computing convenience, it have nothing with the scheme and result. We take the following initial conditions

$$
u(x, y, 0)=2 \operatorname{sech}^{2}\left(x-\frac{\sqrt{2}}{2} y-6\right)
$$

and the exact boundary condition. The KPI equation has the theoretic solution

$$
u(x, y, t)=2 \operatorname{sech}^{2}\left(x-\frac{\sqrt{2}}{2} y-\frac{5}{2} t-6\right)
$$

which represents one line-soliton propagating with the velocity $\frac{5}{2}$ in the direction with the angle of $\tan ^{-1}(\sqrt{2})$ to the positive $x$-axis. We take the test on the domain $[0,40] \times[0,2]$ and choose $\triangle x=0.2, \triangle y=0.1, \triangle t=0.01$. Figure 1 shows the initial condition and Figure 2 shows the numerical solution at time $t=10$. In fact, it propagating 25-unit distant as we indicated.

Next we try two line-soliton interaction.

We take the initial condition

$$
u(x, y, 0)=2 \sum_{i=1}^{2} k_{i}^{2} \operatorname{sech}^{2}\left[k_{i}\left(x+\lambda_{i} y-x_{0, i}\right)\right]
$$

where $k_{1}=1.0, k_{2}=1 / \sqrt{2}, \lambda_{1}=-1 / \sqrt{3}, \lambda_{2}=-1.0$ and $x_{0.1}=6.0, x_{0.2}=11.0$. and the exact boundary condition. The initial condition (4.2) corresponds to two line-solitons, each with amplitude $2 k_{i}^{2}$ placed initially at $x=x_{0, i}$ and moving with velocity $v_{i}=$ $4 k_{i}^{2}-3 \lambda_{i}^{2}$ along the $x$-axis $(i=1,2)$.

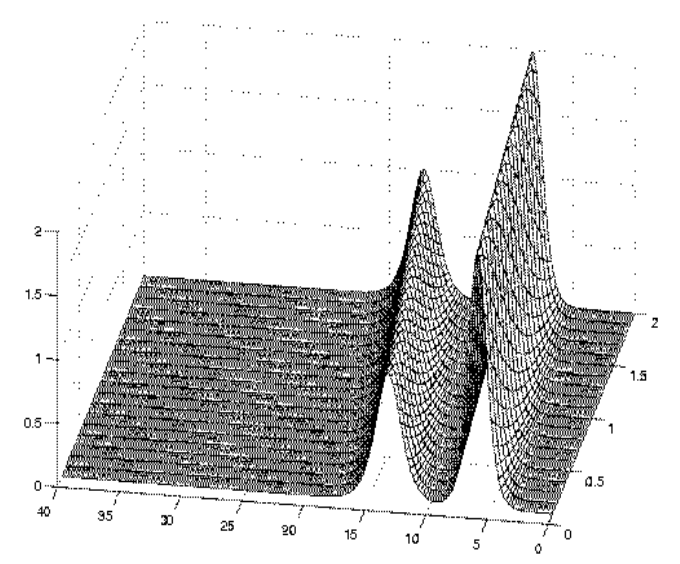

Fig.3: soliton interact at $\mathrm{t}=0$,

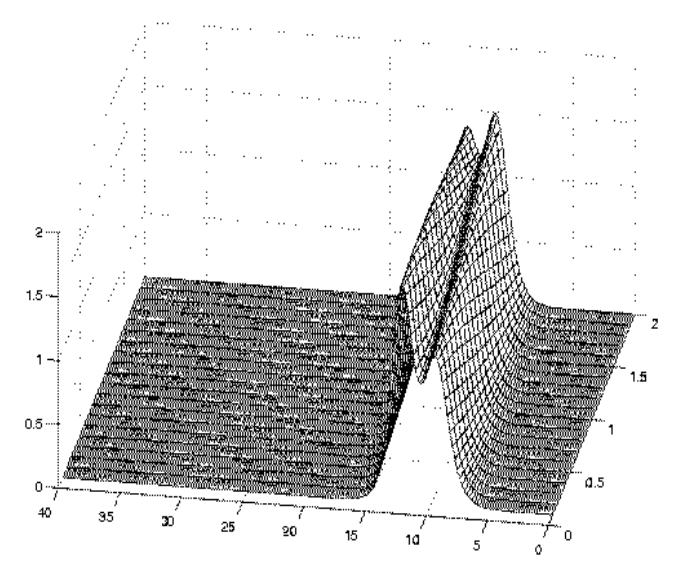

Fig.4: soliton interact at $\mathrm{t}=1.5$,

We carried out the computation on the domain $[0,40] \times[0,2]$, and choose $\triangle x=$ $0.2, \Delta y=0.1, \triangle t=0.01$. The initial condition (4.2) is showed in Figure 3. The larger line-soliton on the right will moves with a velocity 3.0 to the positive $x$-direction and the smaller one on the left will moves with a velocity 1.0 to inverse direction. As time go 


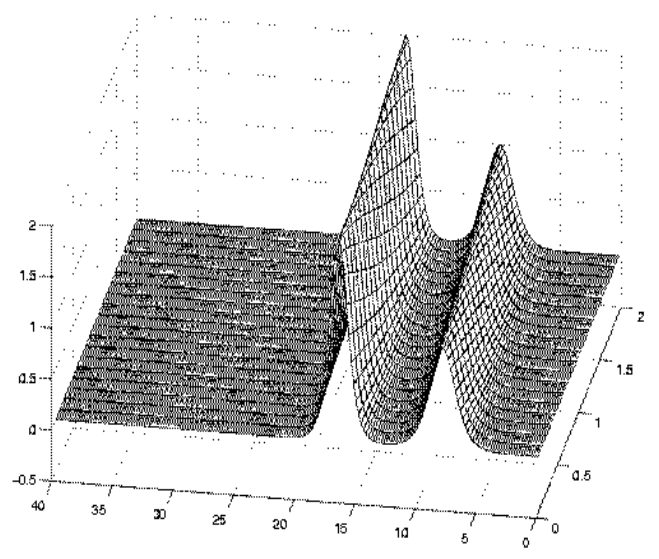

on, they will collide with each other, it is show in Figure 4. Figure 5 shows that the two line-solitons have separated completely after collide and restored their original shape by the $t=3$.

Fig.5: soliton interact at $\mathrm{t}=3$.

We also consider the lump type solitary waves of the KPI equation. The lump type initial condition used for test is

$$
u(x, y, 0)=4 \frac{\left(-\left(x-x_{0}\right)^{2}+\mu^{2}\left(y-y_{0}\right)^{2}+\frac{1}{\mu^{2}}\right)}{\left(\left(x-x_{0}\right)^{2}+\mu^{2}\left(y-y_{0}\right)^{2}+\frac{1}{\mu^{2}}\right)}
$$

where the parameters $\mu^{2}=1.0, x_{0}=10.0, y_{0}=10.0$, and we adopt the exact boundary condition.

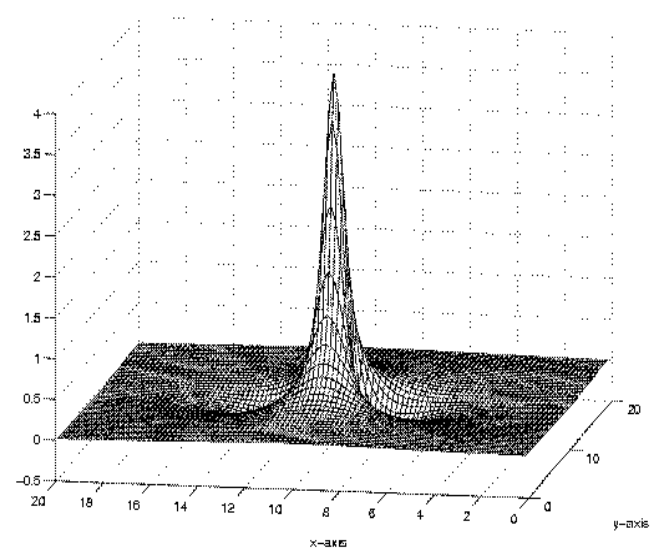

Fig.6: lump type solitary wave at $\mathrm{t}=0$,

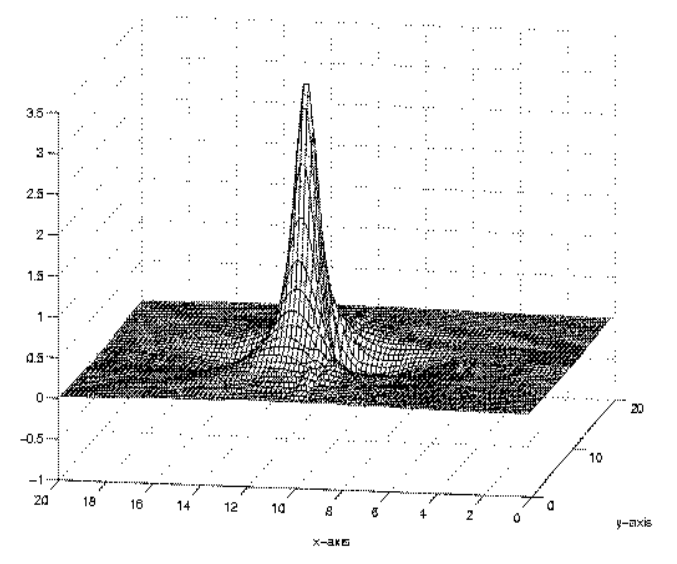

Fig.7: lump type solitary wave at $\mathrm{t}=0.5$.

We compute in a rectangle $[0,20] \times[0,20]$ and choose $\triangle x=0.1, \triangle y=0.2, \triangle t=0.01$. Figure 6 shows the initial condition and figure 7 shows the numerical solution at time $t=0.5$. By the time $t=1$, the result is showed in figure 8. The lump solution of the 
KPI equation can be expressed as

$$
u(x, y, t)=4 \frac{\left(-\left(x-x_{0}-3 \mu^{2} t\right)^{2}+\mu^{2}\left(y-y_{0}\right)^{2}+\frac{1}{\mu^{2}}\right)}{\left(\left(x-x_{0}-3 \mu^{2} t\right)^{2}+\mu^{2}\left(y-y_{0}\right)^{2}+\frac{1}{\mu^{2}}\right)}
$$

According to (4.4), this lump type solitary wave will move to the positive $x$-direction with velocity $3 \mu^{2}$. We can see the moving of the lump solitary wave from the graph.

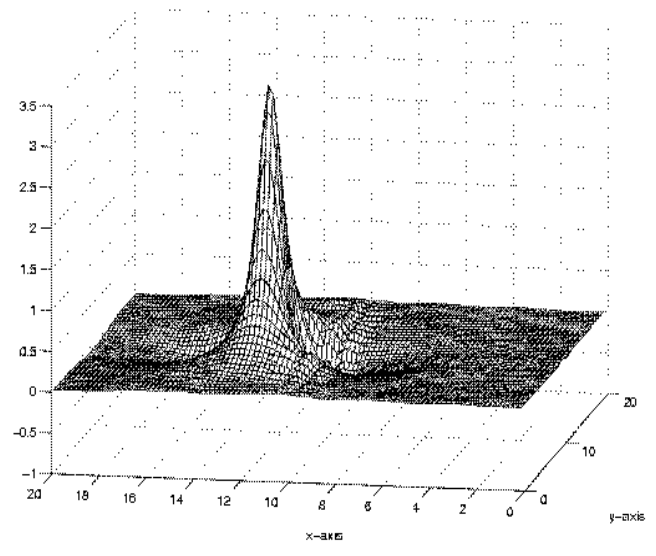

Fig.8: lump type solitary wave at $\mathrm{t}=1$.

A future task is expected to find a proper numerical boundary conditions for collision of the two lump type solitary waves.

\section{References}

[1] V. I. Arnold, Mathematical Methods of Classical Mechanics, Springer-Verlag, NewYork, 1978.

[2] R.W. Atherton and G. M. Homsy, studied in Applied Mathematics, Vol. LIV, No. $1,31,(1975)$

[3] TH. J. Bridges, Math. Proc. Camb. Phil. Soc. 121147 (1997)

[4] TH. J. Bridges, European. J. Mechanics/B, in press

[5] M. J. Gotay, Mechanics, Analysis and Geometry 200 Years after Lagrange M. Francaviglia (Editor) 203 (1991)

[6] J. E. Marsden, and T. S. Ratiu, Introduction to Mechanics and Symmetry, SpringeVerlag, (1997)

[7] J. E. Marsden, G. W. Patrick and S. Shkoller, Comm. Math. Phys. 199, 3515 (1998)

[8] P. J. Olver, Applications of lie groups to differential equations, Springer-Verlag 
[9] S. Reich, JCP, 157, 473 (2000)

[10] P. F. Zhao, M.Z. Qin, J. Phys. A:Math.Gen, 33, 3613 (2000)

[11] P. L. García, A. Pérez-Rendón. Ш, Arch. Rat. Mech. Anal. 43, 101 (1971)

[12] M. J. Ablowitz, P. A. Clarkson, Solitons, Nonlinear Evolution Equations and Inverse Scattering,Cambridge University Press,Cambridge, England, 1991

[13] Bao-Feng feng,Taketomo Mitsui, Journal of Computational and Applied Mathematics 90, 95 (1998)

[14] Shinar Kouranbaeva and Steve Shkoller, A variational approach to second-order multisymplectic field theory, (preprint) 2000

[15] C . Katsis, T . R . Akylas, J. Fluid. Mech.177, 49 (1987)

[16] X. P. Wang, M. J. Ablowitz, H. Segur, Physica D.78, 241 (1994)

[17] A. A. Minzoni, N. F. Smyth, Wave Motion.24, 291(1994)

[18] Th.Donder, Nuov,éd.,Gauthier-Villars,Paris(1935); H.Weyl,Ann.Math(2)36, 607 (1935) 